\title{
Assessment of performance of midwives and pediatricians in preparation for receiving a neonate immediately after birth. A prospective observational study.
}

\author{
Lambros Mpoltsis' ${ }^{1}$ Emmanuel Stamatakis ${ }^{1}$, Theodoros Xanthos ${ }^{2}$, Nicoletta lacovidou ${ }^{3}$, \\ Athanasios Chalkiass', Evangelia Kouskouni ${ }^{4}$, Demetrios Valsamidis ${ }^{1}$ \\ 'Department of Anaesthesiology and Pain Medicine, Alexandra General Hospital, Athens, Greece \\ ${ }^{2}$ Cardiopulmonary Resuscitation, Medical School, European University of Cyprus, Nicosia, Cyprus \\ ${ }^{3}$ Neonatal Division, Department of Pediatrics, Aretaion Hospital, Medical School, National and Kapodistrian \\ University of Athens, Athens, Greece \\ ${ }^{4}$ Department of Anesthesiology, Tzaneio Hospital of Piraeus, Piraeus, Greece \\ ${ }^{5}$ Microbiology laboratory, Aretaieio Hospital, Medical School of the \\ University of Athens, Athens, Greece
}

\section{Correspondence}

Mpoltsis Lambros, Anaesthitist Department, Alexandra Hospital, 80 Vassilisis Sofias Avenue, 11528 Athens Greece, email:boltsis76@hotmail.com

\section{Abstract}

Background. The 2015 European Resuscitation Council (ERC) Guidelines for Resuscitation and support of transition of babies at birth stress the importance of adequate preparation by the healthcare professionals who are going to receive the newborn immediately after birth in order to avoid preventable neonate deterioration. Midwives and pediatricians are the healthcare professionals in the frontline of neonate reception. Methods. Based on the 2015 ERC guidelines we created a 9-item checklist of indispensable actions for correct preparation for neonate reception after vaginal delivery or cesarean section. 78 midwives and 39 pediatricians were included in this prospective observational study. The impact of prior neonate life support training (NLS) on their performance was also assessed. Results. Regarding preparation for neonate reception, participants performed significantly better when the neonate was delivered by vaginal delivery (mean score $7.21 \pm 1.77$ vs $5.45 \pm 1.55$ for cesarean section, $p<0.0005$ ). Furthermore, midwives performed significantly better (performance score 6.88 \pm 1.87 ) than pediatricians even when subgroup analysis was performed for residents $(5.40 \pm 1.59, \mathrm{p}=0.002)$ and consultants $(5.46 \pm 1.47$, $\mathrm{p}=0.002)$. Previous NLS training resulted in significantly higher performance scores $(6.57 \pm 1.81$ vs $5.18 \pm 1.91$ for no NLS training, $\mathrm{p}=0.004$ ). Conclusions. In the present study midwives performed better than consultant and resident pediatricians in preparing for receiving a neonate immediately after birth 
and neonatal life support training led to significantly better performance when compared to particiants with no prior NLS training. To our knowledge, this is the first study to assess these skills in midwives and pediatricians.

Key words: Midwife, pediatrician, neonate, resuscitation, preparation

\section{Introduction}

It is well established that only a small percentage of infants require resuscitation at birth ${ }^{1-3}$. However, they all need a specific series of actions and a standard level of care in order not to rapidly deteriorate due to their limited reserves ${ }^{3,4}$. The necessary preparations to be undertaken are currently described in the 2015 European Resuscitation Council (ERC) Guidelines for Resuscitation and support of transition of babies at birth ${ }^{4}$. These Guidelines stress the importance of adequate preparation by the healthcare professionals who are going to receive the newborn and even state that resuscitation at birth is a predictable event and hence it is possible to prepare the environment and the equipment before delivery.

The need for training of healthcare practitioners in neonatal resuscitation and is significant impact on infant outcome have been well recognized and over the last 20 years several programs have been implemented in both developed and in low resource countries with important results ${ }^{5-12}$. The diminishing numbers of available doctors further stress the importance of the role of midwives and neonatal nurse practitioners as the frontline of neonatal resuscitation $^{10,13}$. In Greece, NLS is not mandatory and it is not part of the midwifery academic curriculum.

Given the importance of correct preparation of the environment and the equipment for preventing neonatal deterioration, the present study aimed to assess the level of performance of midwives and doctors (consultants and residents) who are involved in preparing for receiving infants after delivery and whether their level of NLS training plays a role in their performance, in a large university maternity hospital in Greece, using a 9-item checklist.

\section{Materials and Methods}

From June 2014 to September 2015 following "Alexandra" General Hospital of Athens ethics committee approval (12/05/2014, protocol $\mathrm{Nr} 56 /$ 2014) and participants' written informed consent, 117 midwives and pediatricians (residents and consultants) were included in this prospective observational study. Demographic data (including whether the participants had previous ERC-NLS training) were recorded for all participants as well as the mode of delivery (vaginal birth or cesarean section). In order to avoid experience bias, only healthcare professionals with a work experience of more than a year in our hospital were included in the study. Furthermore, in order to avoid bias related to a theoretically more meticulous preparation by the participants, instrumental deliveries and category I and II cesarean section deliveries were excluded from the study. Hence, a homogenized setting of deliveries was created and participants had to prepare for a non-emergent delivery.

Based on the 2015 European Resuscitation Council Guidelines for Resuscitation and support of transition of babies at birth ${ }^{4}, 9$ distinct steps to be assessed (using Yes/No assessment forms) where prepared by the investigators (Table 1). These steps were regarding correct preparation for receiving the neonate as per ERC guidelines. This list of actions was also assessed for importance, relevance and clarity of content by 4 local neonatal life support (NLS) instructors and a NLS course director in Athens before the beginning of the study. The per- 
formance of the study participants was recorded by one of the investigators on the 9-item checklist specifically created for the study and a performance score was given for each one of them depending on how many steps they had performed correctly (for example if one participant had performed correctly 7 out 9 steps, his respective score was 7).

\section{Statistical analysis}

Continuous data are presented as means \pm standard deviation (SD). The Kolmogorov-Smirnov test was utilized for normality analysis of the parameters. Categorical data are presented either as median and range or as frequencies and percentages. Demographic categorical data comparison regarding overall performance was performed using Independent samples t-test and ANOVA, whereas quantitative demographic variables where assessed using Pearson's correlation coefficient. All demographic variables with a $\mathrm{p}$ value $<0.2$ during one-dimensional analysis were included in multidimensional analysis. The correlation between dependent factors and demo- graphic variables was assessed using multiple linear regression with enter method. All tests were twosided. The level of statistical significance for all analyses was set as $\mathrm{p}<0.05$. The SPSS statistical package (SPSS, Chicago ILL Version 17 for Windows) was used for all analyses.

\section{Results}

Demographic characteristics of the study participants are shown in Table 2. The overall performance of the participants is presented in Table 3 and Figure 1. Regarding preparation for neonate reception, participants performed significantly better when the neonate was delivered by vaginal delivery (mean score $7.21 \pm 1.77$ vs $5.45 \pm 1.55$ for cesarean section, $\mathrm{p}<0.0005$ ). Furthermore, midwives performed significantly better (performance score $6.88 \pm 1.87$ ) than pediatricians even when subgroup analysis was performed for residents $(5.40 \pm 1.59, \mathrm{p}=0.002)$ and consultants $(5.46 \pm 1.47, \mathrm{p}=0.002)$. Previous NLS training resulted in significantly higher performance scores (6.57 \pm 1.81 vs $5.18 \pm 1.91$ for no NLS training,

Table 1. Neonatal resuscitation preparation algorithm

Are there usually enough hot towels and a bag for heat protection of very premature infants in the recovery kit?

Does the material for resuscitation check and all work properly?

Is it assured that oxygen (02) is available if needed?

Is it checked if a pulse oximeter and neonatal sensor are available if needed? catheter type and size? Are there different catheter sizes?

Is suction controlled if it works and if properly adjusted with the correct

Is there a Guedel, Laryngoscope, and Stethoscope?

Are there any recovery drugs (adrenaline, sodium carbonate, dextrose), and infusion fluids $(0.9 \% \mathrm{NaCl}, 10 \%$ Dextrose $)$ ?

Is the clock checked?

Is check-up for transport incubator readiness if transport is needed?

$\%$

0,0 100,0 0,0 100,0 5,1

94,9 50,4 49,6 22,2 77,8 47,0 53,0 51,3

48,7

69,2

30,8

17,9

82,1 
$\mathrm{p}=0.004$ ). After excluding the mode of delivery because of high correlation with the other independent variables, multiple linear regression-enter method analysis revealed that pediatricians had an inferior performance by 1.5 performance score units $(p<0.0005)$ in preparing for neonate reception when compared to midwives. NLS training resulted in a rise of 1.9 units in healthcare professionals' performance score $(\mathrm{p}<0.0005)$ when compared to those with no NLS training. No differences were observed between gender and performance scores $(7.00 \pm 1.71$ for men and $6.3 \pm 1.90$ for women, $\mathrm{p}=0.321)$. The same applied for age $(\mathrm{p}=0.485)$.

\section{Discussion}

Neonatal deaths have been reduced worldwide the last two decades ${ }^{14}$. However the causes and rates of neonatal deaths greatly vary between continents and countries ${ }^{14-16}$. Whereas the causes of neonatal deaths in less developed countries depend principally on the lack of access to healthcare infrastructures, in developed countries the main focus is given to healthcare education, in reducing morbidity and in prevention by early recognition of neonate deterioration $^{14-16}$. The title of ERC 2015 Guidelines ${ }^{4}$ (Re- suscitation and support of transition of babies at birth) is very characteristic. However, even in developed countries there are differences between the academic curricula of the healthcare professionals who will first receive a baby at birth, i.e., midwives and pediatricians ${ }^{10,17-19}$. In Greece, NLS training does not make part of the academic training either for midwives or for doctors or pediatricians during their internship. Therefore, in the present study we aimed to assess whether NLS training would provide better adherence to ERC Guidelines and hence improved performance in preparing for receiving a baby at birth and whether the different academic training (midwife versus doctor) could also affect performance. To our knowledge this is the first study assessing the preparation steps followed by healthcare practitioners according to ERC guidelines prior to receiving a baby after birth and hence the direct comparison of the study's results with those of other studies is difficult. However, there are studies in the international literature comparing resuscitations skills of neonatal nurse practitioners or midwives with doctors.

In our study, midwives outperformed resident and consultant pediatricians in the correct preparation

Table 2. Participants demographics

\begin{tabular}{|c|c|c|c|c|}
\hline & & & Average & TA p-value \\
\hline \multirow[t]{2}{*}{ Birth } & Physical & 7,21 & 1,77 & $<0.0005$ \\
\hline & Caesarean & 5,45 & 1,55 & \\
\hline \multirow[t]{2}{*}{ Sex } & Man & 7,00 & 1,71 & 0.321 \\
\hline & Woman & 6,30 & 1,90 & \\
\hline \multirow[t]{2}{*}{ Children } & No & 6,35 & 1,81 & 0.914 \\
\hline & Yes & 6,39 & 2,05 & \\
\hline \multirow[t]{4}{*}{ Education } & Midwife & 6,88 & 1,87 & 0.002 \\
\hline & Midwife+MSc & 6,75 & 1,96 & \\
\hline & Pediatrician-Specialty & $5,40 \alpha, \beta$ & 1,59 & \\
\hline & Pediatrician & $5,46 \alpha, \beta$ & 1,47 & \\
\hline \multirow[t]{2}{*}{ Marital status } & Unmarried & 6,41 & 1,83 & 0.754 \\
\hline & Married & 6,29 & 2,00 & \\
\hline Has he been taught-informed about $\mathrm{t}$ & No & 5,18 & 1,91 & 0.004 \\
\hline he algorithm of N.L.S the invisible? & Yes & 6,57 & 1,81 & \\
\hline
\end{tabular}


for receiving a neonate immediately after birth. This result is in accordance with what was observed in other studies where it was reported that babies resuscitated by neonatal nurse practitioner teams were resuscitated at least as effectively as and even better than those who were received by medically led teams ${ }^{1,12,19-21}$. On the other hand there are reports in the literature that non-medical staff did not perform as well as medical staff in their overall completeness of care; however this was done at acceptable standards ${ }^{13}$.

Table 3. Postpartum course

\begin{tabular}{llll}
\hline & & N & \% \\
Does the watch start? & no & 68 & 58,1 \\
& yes & 49 & 41,9 \\
Does the child dry? & no & 5 & 4,3 \\
Are wet towels removed? & yes & 112 & 95,7 \\
Is the child covered? & no & 4 & 3,4 \\
& yes & 113 & 96,6 \\
Is color, tone, breathing & no & 15 & 12,8 \\
and heart rate estimated? & yes & 102 & 87,2 \\
& no & 1 & 0,9 \\
& yes & 116 & 99,1
\end{tabular}

The better overall performance of midwives when compared to doctors observed in our study could be attributed to 2 factors: different education structure and overall experience in receiving neonates. In Greece, neonate examination is a mandatory part of the undergraduate academic midwife curriculum and the evaluation is clinically based. On the other hand, pediatric residents rely on reading, training and self-directed learning throughout residency guided by their consultants. Furthermore, pediatricians, unlike for cesarean sections, are only called for complicated vaginal deliveries, fact that leads to a smaller number of cases treated by pediatricians leading over time to a higher number of infants received by midwives which profit in experience more rapidly by this way.
In our study, previous NLS training resulted in significantly higher performance scores of healthcare professionals receiving neonates after birth $(6.57 \pm 1.81$ vs $5.18 \pm 1.91$ for no NLS training, $\mathrm{p}=0.004$ ). It has already been reported that a focused neonatal resuscitation course in midwives ${ }^{10}$ leads to improved neonate management. Neonatal resuscitation courses have been offered to midwives in several countries over the last 2 decades $^{7,8,10}$ but in a very few cases a structured course has been embedded in the midwife academic curriculum and that is also the case in Greece. However, there are several studies reporting that structured simulation training not only improves the healthcare professionals' performance, but it also increases their self-confidence and competencies ${ }^{7,22-24}$.

In the present study participants performed significantly better when preparing to receive a neonate after vaginal delivery than after a cesarean section. This is a puzzling finding as the opposite should probably have been anticipated. Perhaps because the frontline healthcare responders more often encounter babies delivered vaginally (vaginal deliveries outnumber cesarean sections) they are accustomed and feel more at ease with that procedure, following therefore more easily their preparation routine. That could especially apply for midwives, who are the

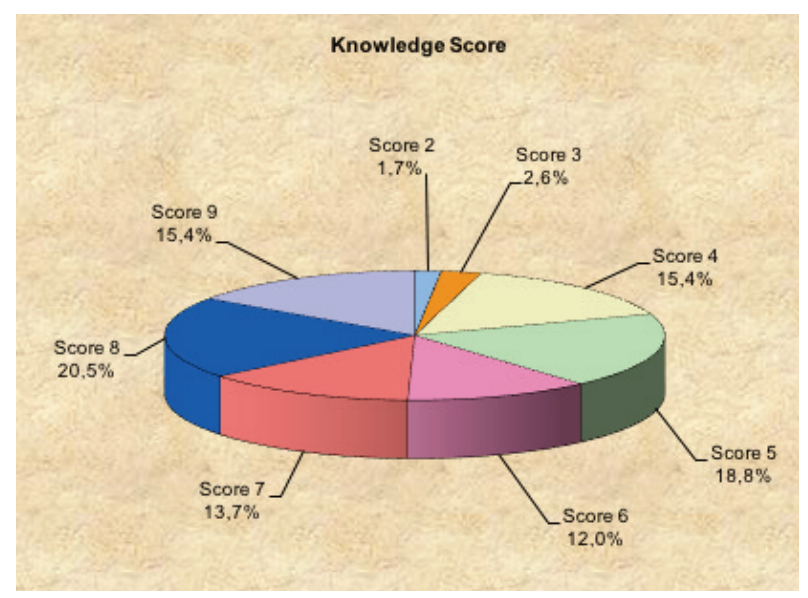

Figure 1. Participants knowledge score 
healthcare providers that are more implicated in vaginal labor. Furthermore, cesarean section as a "not normal delivery" and by its own nature as an operation may constitute an important stress factor for midwives and pediatricians, therefore rendering error easier to occur.

The aforementioned results underline the paramount importance of focused training and assessment of clinical skills. Participants with NLS training performed significantly better than those who had never attended such a course. However, even NLS training did not prove to be enough and no more than $15 \%$ of overall participants succeeded in performing all of the preparations steps. It is well known that only training and assessment cannot prevent human error from occurring. Education and training in human factors (team working, situation awareness, leadership) in critical incidents is mandatory. However, simulation training only, may not be enough for improving preparation to initiate newborn resuscitation ${ }^{25}$. Checklists (as the WHO surgical checklist ${ }^{26}$ should probably be implemented in this setting also as ILCOR guidelines clearly recommend ${ }^{3}$. The 9-item checklist used in the study could provide a base for a future checklist for use in our hospital and its validity will be assessed in a subsequent study.

The present study has several limitations. Despite the fact that it has been undertaken in the largest maternity university hospital in Greece, the number of the participants was relatively small in order to deduct safe results. However, it is extremely difficult to obtain larger numbers of midwives and pediatricians in single center. Another limitation of the study was that participants were aware that their actions were recorded and evaluated. This could probably constitute a bias as they may have been more attentive and hence may have performed better than in routine practice.

In conclusion, in the present study midwives per- formed better than consultant and resident pediatricians in preparing for receiving a neonate immediately after birth and neonatal life support training led to significantly better performance when compared to participants with no prior NLS training. To our knowledge, this is the first study to assess these skills in midwives and pediatricians and it adds some evidence to the existing literature concerning midwife and pediatrician training.

\section{References}

1. Aziz K, Chadwick M, Baker M, Andrews W. Anteand intra-partum factors that predict increased need for neonatal resuscitation. Resuscitation 2008;79:444-52.10.

2. Palme-Kilander C. Methods of resuscitation in low-Apgar-score newborn infants-a national survey. Acta Paediatr 1992;81:739-44.

3. Wyckoff MH, Aziz K, Escobedo MB, Kapadia VS, Kattwinkel J, Perlman JM, Simon WM, Weiner GM, Zaichkin JG. Part 13: Neonatal Resuscitation: 2015 American Heart Association Guidelines Update for Cardiopulmonary Resuscitation and Emergency Cardiovascular Care. Circulation. 2015;132(18 Suppl 2):S543-60.

4. Wyllie J, Bruinenberg J, Roehr CC, Rüdiger M, Trevisanuto D, Urlesberger B. European Resuscitation Council Guidelines for Resuscitation 2015: Section 7. Resuscitation and support of transition of babies at birth. Resuscitation. 2015; 95:249-63.

5. Lee AC, Cousens S, Wall SN, Niermeyer S, Darmstadt GL, Carlo WA, Keenan WJ, Bhutta ZA, Gill C, Lawn JE. Neonatal resuscitation and immediate newborn assessment and stimulation for the prevention of neonatal deaths: a systematic review, meta-analysis and Delphi estimation of mortality effect. BMC Public Health. 2011;11 Suppl 3:S12. doi: 10.1186/1471-2458-11-S3S12.

6. Wall SN, Lee AC, Niermeyer S, English M, Keenan 
WJ, Carlo W, Bhutta ZA, Bang A, Narayanan I, Ariawan I, Lawn JE. Neonatal resuscitation in lowresource settings: what, who, and how to overcome challenges to scale up? Int J Gynaecol Obstet. 2009;107 Suppl 1:S47-62, S63-4.

7. Agrawal N, Kumar S, Balasubramaniam SM, Bhargava S, Sinha P, Bakshi B, Sood B. Effectiveness of virtual classroom training in improving the knowledge and key maternal neonatal health skills of general nurse midwifery students in Bihar, India: A pre- and post-intervention study. Nurse Educ Today. 2016;36:293-7.

8. Bang A, Bellad R, Gisore P, Hibberd P, Patel A, Goudar S, Esamai F, Goco N, Meleth S, Derman RJ, Liechty EA, McClure E, Carlo WA, Wright LL. Implementation and evaluation of the Helping $\mathrm{Ba}$ bies Breathe curriculum in three resource limited settings: does Helping Babies Breathe save lives? A study protocol. BMC Pregnancy Childbirth. 2014;14:116.

9. Lawn JE, Kinney M, Lee AC, Chopra M, Donnay F, Paul VK, Bhutta ZA, Bateman M, Darmstadt GL. Reducing intrapartum-related deaths and disability: can the health system deliver? Int J Gynaecol Obstet. 2009;107 Suppl 1:S123-40, S140-2.

10. Singh J, Santosh S, Wyllie JP, Mellon A. Effects of a course in neonatal resuscitation--evaluation of an educational intervention on the standard of neonatal resuscitation. Resuscitation. 2006;68:385-9.

11. Singhal N, McMillan DD, Yee WH, Akierman AR, Yee YJ. Evaluation of the effectiveness of the standardized neonatal resuscitation program. J Perinatol. 2001;21:388-92.

12. Aubrey WR, Yoxall CW. Evaluation of the role of the neonatal nurse practitioner in resuscitation of preterm infants at birth. Arch Dis Child Fetal Neonatal Ed. 2001;85:F96-9.

13. Woods L. Evaluating the clinical effectiveness of neonatal nurse practitioners: an exploratory study. J Clin Nurs. 2006;15:35-44.

14. http://www.who.int/mediacentre/factsheets /fs290/en/

15. Oestergaard MZ, Inoue M, Yoshida S, Mahanani WR, Gore FM, Simon C, Joy E, Lawn JE, Colin Douglas $\mathrm{M}$, on behalf of the United Nations Interagency Group for Child Mortality Estimation and the Child Health Epidemiology Reference Group Neonatal mortality levels for 193 countries in 2009 with trends since 1990: a systematic analysis of progress, projections, and priorities. PLoS Med 2011, 8(8):e1001080.

16. Rajaratnam JK, Marcus JR, Flaxman AD, Wang H, L-R A, Dwyer L, Lopex AD, Murray CJL: Neonatal, postneonatal, childhood, and under-5-mortality for 187 countries, 1970-2010; a systematic analysis of progress towards Millennium Development Goal 4. Lancet 2010, 375:1988-2008.

17. Singhal N, McMillan DD, Yee WH, et al. Evaluation of the effectiveness of the standardized neonatal resuscitation program. J Perinatol 2001;21:388-92.

18. Neonatal Resuscitation Program. Textbook of neonatal resuscitation. Dallas: American Heart Association \& American Academy of Paediatrics, 2001.

19. Chan LC, Hey E. Can all neonatal resuscitation be managed by nurse practitioners? Arch Dis Child Fetal Neonatal Ed. 2006;91:F52-5.

20. Neal D, Stewart D, Grant CC. Nurse-led newborn resuscitation in an urban neonatal unit. Acta Paediatr. 2008;97:1620-4.

21. Lee TW, Skelton RE, Skene C. Routine neonatal examination: effectiveness of trainee paediatrician compared with advanced neonatal nurse practitioner. Arch Dis Child Fetal Neonatal Ed. 2001;85:F100-4

22. Halamek LP. The simulated delivery-room environment as the future modality for acquiring and maintaining skills in fetal and neonatal resusci- 
tation. Semin Fetal Neonatal Med. 2008;13:44853.

23. Ten Eyck RP, Tews M, Ballester JM. Improved medical student satisfaction and test performance with a simulation-based emergency medicine curriculum: a randomized controlled trial. Ann Emerg Med. 2009;54:684-91.

24. Steadman RH, Coates WC, Huang YM, Matevosian R, Larmon BR, McCullough L, Ariel D. Simulationbased training is superior to problem-based learning for the acquisition of critical assessment and management skills. Crit Care Med. 2006;34:151-7.
25. Bull A, Sweet L. Midwifery students receiving the newborn at birth: A pilot study of the impact of structured training in neonatal resuscitation. Nurse Educ Pract. 2015;15:387-92.

26. Mahajan RP. The WHO surgical checklist. Best Pract Res Clin Anaesthesiol. 2011;25:161-8.

Received 2-5-2018

Revised 27-5-2018

Accepted 12-6-2018 